\title{
Differential effects of syntactic and semantic processing on the subregions of Broca's area
}

\author{
Sharlene D. Newman*, Marcel Adam Just, Timothy A. Keller, Jennifer Roth, \\ Patricia A. Carpenter \\ Center for Cognitive Brain Imaging, Department of Psychology, Carnegie Mellon University, Baker Hall 327, 5000 Forbes Ave., \\ Pittsburgh, PA 15213, USA
}

Accepted 20 November 2002

\begin{abstract}
This study attempts to specify the contribution of two subregions of Broca's area during syntactic and semantic processing of sentences by examining brain activation in a grammaticality judgment task. The processing of two types of ungrammatical sentences was examined. One type leaves the thematic interpretation generally unaffected, by violating the noun-verb agreement in number, while the other type introduces an extraneous verb, which cannot be incorporated into the developing thematic structure. Pars triangularis was more sensitive to the extra verb violation, whereas pars opercularis was more sensitive to the noun-verb agreement violation. The current study adds to the growing literature that suggests there are separable functional subregions of Broca's area, with pars triangularis more involved in thematic processing and pars opercularis more involved in syntactic processing. The posterior left temporal area was also involved in both types of processing.
\end{abstract}

(C) 2002 Elsevier Science B.V. All rights reserved.

Theme: Neural basis of behaviour

Topic: Cognition

Keywords: cerebral cortex; fMRI; Grammar; Syntax; Semantics; Sentence comprehension

\section{Introduction}

The neural basis of syntactic processing has been examined through lesion studies and recently with the use of functional neuroimaging. Evidence from these studies suggests that syntactic processes are supported, at least in part, by the left inferior frontal gyrus, or Broca's area $[7-10,13,17,19,22,23,41]$. (Note that the posterior left temporal area was also implicated in most studies, so the inferior frontal area is not the sole seat of syntactic processing). While these studies are all consistent with Broca's area being involved in syntactic processing, they fail to converge on a single region within the inferior frontal cortex. For example, a plausibility judgment task contrasted syntactically simpler right-branching sentences

\footnotetext{
*Corresponding author. Tel.: +1-412-268-3784; fax: +1-412-2682804.

E-mail address: snewman@andrew.cmu.edu (S.D. Newman).
}

with more complex center-embedded sentences and found increased blood flow in the left pars opercularis during the more complex sentences [41]. These findings were generally replicated in another study by the same group with auditory presentation in which subjects judged the plausibility of cleft-subject and more complex cleft-object sentences, except that the activation was in pars triangularis rather than pars opercularis [8]. These studies clearly implicate the inferior frontal gyrus in syntactic processing, although there is no convergence on a particular location, perhaps in part because of the differences in particular tasks and image subtractions.

Recent studies have suggested that the processing of the frontal operculum and the pars triangularis are different. In fact neuroimaging studies examining single word processing (e.g. verb generation tasks) have found that the pars triangularis was involved in semantic processing $[7,8,10,18-20]$. This suggests that the activation of the pars triangularis during sentence comprehension may also 
be related to semantic processing. The term semantic processing is used here to denote the processing of sentence meaning, which includes thematic role assignment as well as word meaning. In a study comparing syntax and script processing, patients whose lesions were located in pars triangularis and extending anteriorly were found to have significant difficulty with the script task (i.e. producing a logical story narrative from a list of actions) [39]. Conversely, their performance on a syntactic task was relatively unimpaired. These results suggest that the processing of pars triangularis may involve not only semantic processing at the level of individual word meanings, but it may also be involved in processing actions and their arguments, such as agents and patients. This is the essence of thematic processing.

There is also growing evidence to suggest that pars opercularis is involved in syntactic level processing $[8,13,17,19,23,41]$. For example, in one of the studies mentioned above [39], patients with lesions confined to the frontal operculum and extending posteriorly had significant difficulty when performing a syntax task (i.e. producing a grammatically correct sentence by assembling a list of phrases into a sensible order), while their performance on the script task was relatively unimpaired.

Syntactic and semantic information have to be integrated at some point, and the integration of these two types of information makes it difficult to study them separately. This is because when the syntactic structure is disrupted, a potential thematic anomaly can also be produced. For example, in the expression, 'all the eaten have chickens snakes,' the anomalous syntactic structure also wreaks havoc with the thematic interpretation. The interplay between semantics and syntax can also be observed in the processing of complex sentence structures such as objectrelative sentences. It has been hypothesized that what makes processing object-relative sentences more difficult than subject-relative sentences is that maintaining the head of the clause in a buffer creates a lag in integrating the material into the developing semantic representation $[3,8]$. This implies that object-relative sentences not only create an increased processing load for the cortical regions responsible for syntactic processing but also for those responsible for semantic processing. Therefore, one explanation for the inconsistency in the activation location with Broca's area (pars triangularis vs. pars opercularis) during complex syntactic processing is this interaction between syntax and semantics: complex syntactic processing is likely to result in complex semantic processing.

Because semantic and syntactic processes are so intimately intertwined, it is extremely difficult to examine one alone. However, in the current study, we attempt to specify the contribution of two sub-regions of Broca's area during syntactic and semantic processing by examining relative differences in their responses to two types of grammatical violations. Therefore, the processing centers related to both semantics and syntax are expected to be involved in the

processing of both types of violations, but to differing levels. The first type leaves the semantic interpretation relatively unaffected, by manipulating the noun-verb agreement, such as 'The lady praises the sister and meet the artist in the night.' The second type of grammatical violation was designed to differentially affect the semantic interpretation by adding an extra verb, such as 'The woman thanked the barber and paid the receptionist knew at the desk.' It has been postulated that the lexical content of verbs entails their predicate argument structure and establishes thematic roles in sentences [12,37]. If this is the case, then adding an extra verb will disrupt the thematic/ semantic processing. In addition, the ERP N400 response has been suggested to indicate how easily a word can be integrated into the semantic representation of a sentence $[5,21,33]$, and it has often been observed during the processing of semantic anomalies [30,37].

\section{Materials and methods}

\subsection{Participants}

Participants were thirteen right-handed native English speakers from the Carnegie Mellon University community. All of the participants gave informed consent that was approved by the University of Pittsburgh and Carnegie Mellon Institutional Review Boards.

\subsection{Experimental paradigm}

There are two types of sentences: conjoined active and object relative. There are two types of ungrammaticalities: noun-verb agreement and extra-verb. Hence, there were four conditions (four epoch types). Examples of the two problem types are below:

Noun-verb agreement
Conjoined active

Object relative

Extra verb

Conjoined active

Object relative

Each epoch contained five sentences, three of which
The lady praises the sister and meet the artist in the night.

The waitress that the lawyer interrupts notice the commander on the stairs.

The coach watched the poet and told the visitor took in the evening.

The duke that the seamstress forgave walked the baby took down the hall. 
contained a grammatical violation. Within each epoch all of the sentences were of the same structure (either conjoined active or object relative) and the ungrammatical sentences all contained the same type of violation. A total of 100 sentences were presented (15 for each of the four conditions and 40 grammatically correct sentences, distractor items, 20 conjoined active and 20 object-relative). The nouns and verbs used in the sentences were high-frequency words from the Kucera \& Francis word frequency inventory. During the test session, the sentences were projected onto a transparent screen suspended from the upper surface of the scanner bore. Both the sentence and the response alternatives were displayed on the screen simultaneously and the participants were to judge whether the sentence was grammatically correct by pressing the appropriate response button. The grammaticality judgment response terminated the presentation of one sentence and initiated the presentation of the next one. Between each epoch was a 6 second rest period. The images collected during the rest periods and the first $6 \mathrm{~s}$ of each epoch were discarded to accommodate the rise and fall of the hemodynamic response [1]. In addition, four 24-s fixations were evenly distributed to obtain a control baseline measure with which to compare experimental conditions.

\section{3. fMRI procedure}

The study was conducted on a GE 3.0 Tesla scanner used in conjunction with a commercial birdcage, quadrature-drive radio-frequency whole-head coil. Fourteen oblique-axial images were selected to maximize the coverage of the parietal, temporal, and frontal lobes. The images were collected using a gradient echo, resonant echo planar pulse sequence, with $\mathrm{TR}=3000 \mathrm{~ms}, \mathrm{TE}=50 \mathrm{~ms}$, flip angle $=90^{\circ}$, and a $128 \times 64$ acquisition matrix with a voxel size of $3.125 \times 3.125 \times 5 \mathrm{~mm}$ with a $1-\mathrm{mm}$ gap.

The means of the images corresponding to each of the functional slices were registered to a high-resolution, T1weighted structural volume scan of each participant. This volume scan was constructed from 124 3D SPGR axial images that were collected with $\mathrm{TR}=25 \mathrm{~ms}, \mathrm{TE}=4 \mathrm{~ms}, 40^{\circ}$ flip-angle, and a $24 \times 18-\mathrm{cm} \mathrm{FOV}$, resulting in $0.9375 \times$ $0.9375 \times 1.5 \mathrm{~mm}$ voxels.

\subsection{Anatomical regions of interest}

To compare the amount of activation in a given area across experimental conditions, anatomically-defined ROIs were drawn on the structural images, without the activation overlaid, for each participant using the parcellation scheme described by Rademacher et al. [36] and further refined by Caviness et al. [11] (Fig. 1). In order to examine how the type of grammatical violation and the sentence type affected the volume and amplitude of the activation in each of these regions, it was important to use an a priori, independent method of defining the ROIs.

ROIs for the cortical areas known to activate in language tasks were defined. There were two separate ROIs in the inferior frontal gyrus, the pars opercularis ROI (F3o; BA 44) and the pars triangularis ROI (F3t; BA 45/47). The temporal ROI included both the superior and middle temporal gyri (T1a, T1p, T2a, T2p, TO2; BA 22/21) (The superior and middle temporal gyri were combined into one ROI because previous studies of language processing have often found activation centered in the superior temporal sulcus between them [25]). The intraparietal ROI was defined as the main branch of the intraparietal sulcus (two functional voxels wide) excluding any medial or lateral branches. The extrastriate ROI consisted of both inferior and superior extrastriate cortex (TOF and TF). There were two middle frontal ROIs. The dorsolateral prefrontal cortex ROI was defined as the middle frontal gyrus (F2), but excluding the anterior bank (two voxels wide) of the pre-central sulcus, which was defined as the frontal eye fields ROI. The ROIs were drawn separately for the left and right hemispheres. A member of the staff defined the ROIs for each participant after extensive training on the parcellation scheme. The inter-rater reliability of this ROI-

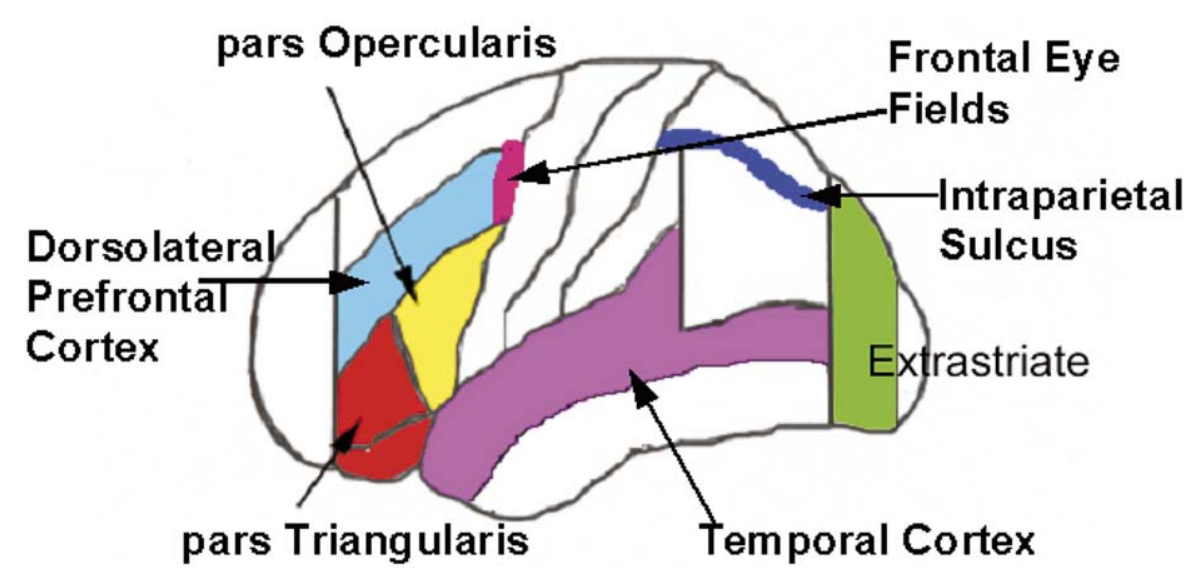

Fig. 1. A schematic of the regions of interest. 
defining procedure between two trained staff members was previously evaluated for four ROIs in two participants in another study. The reliability measure was obtained by dividing the size of the set of voxels that overlapped between the two raters by the mean of their two set sizes. The resulting eight reliability measures were in the 78 $91 \%$ range, with a mean of $84 \%$. This reliability is exceptional in that it is as high as the reliability reported by the developers of the parcellation scheme.

\section{5. fMRI data analysis}

fMRI-measured activation was quantified in two ways: For each condition, we determined the mean number of voxels within each region of interest (ROI) with activation levels higher than in the baseline condition using a $t$-test with a threshold of $t>5.0$ (which is more conservative than the Bonferroni correction for $P<0.01)$. Second, for the activated voxels within each ROI, we calculated the sum percent increase in their signal intensity (SSI) relative to the baseline. (This measure is an integral of the mean signal change and number of active voxels). We used analysis of variance (ANOVA) tests to compare the effects of the two types of grammatical violation and sentence types.

Image preprocessing corrected for head motion and signal drift using FIASCO $[16,26]$. The fMRI procedure relies on BOLD contrast (blood oxygenation level-dependent) to measure the oxygen level in the microvasculature supporting neuronal activity. Two steps were taken to insure that the fMRI-measured activation was due to changes in cortical micro-vascular activity rather than changes in the blood-flow rate of larger vessels. First, the activation maps that were defined by the ROIs corresponded to cortical tissue and not to the spaces normally occupied by cerebrospinal fluid or large blood vessels. The distribution of activation was thus confined to a discrete volume in the image space that did not correspond to the known drainage pattern of large veins. Second, to reduce the influence of large blood vessels, any voxel that showed an excessively large percentage change in signal intensity (greater than $6.2 \%$ ) was excluded from the analyses. This procedure usually results in the exclusion of about $1 \%$ of voxels in all ROIs.

\section{Results}

\subsection{Behavioral results}

The data were analyzed using a $2 \times 2$ factorial ANOVA, with sentence type and type of violation as within subject variables. There were no statistically significant differences in the error rates or response times during the processing of object-relative sentences compared to conjoined active sentences, shown in Fig. 2. Furthermore, there were no

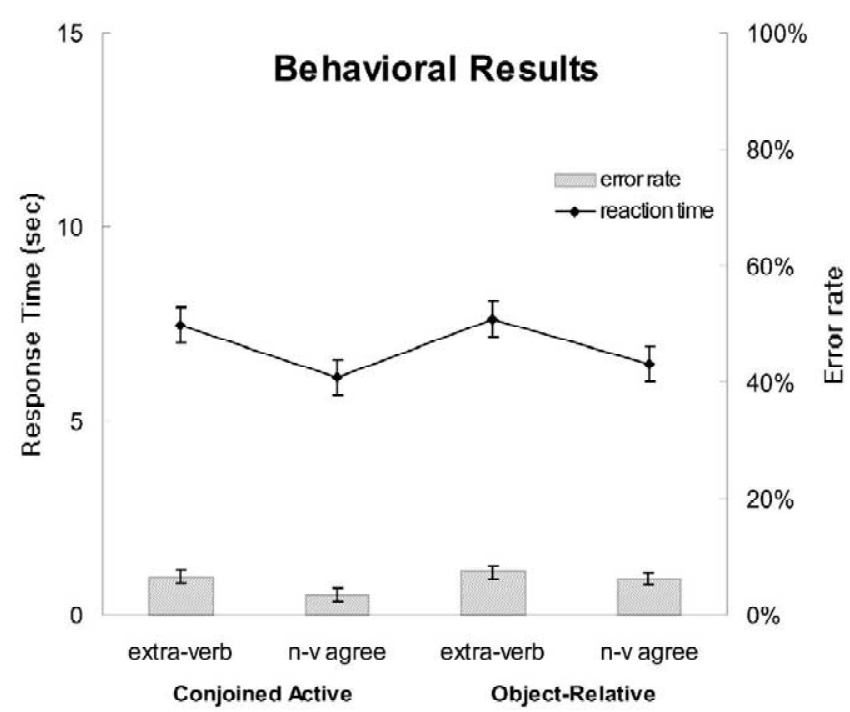

Fig. 2. The error rates and response times for each condition. Error bars represent $95 \%$ confidence intervals based on the pooled Mse from the corresponding ANOVA (Loftus and Mason, 1994).

significant differences in the response times (although there was a trend) or the error rates when comparing the extraverb condition and the noun-verb agreement condition [response time: $F(1,12)=3.77, P>0.07$; error: $F<1$ ]. The absence of a reliable sentence complexity effect during grammaticality judgment stands in contrast to the oftenreported presence of such effects when the task requires comprehension of the sentence content $[25,28]$.

\section{2. fMRI results}

Both violation type and sentence complexity modulated the volume of cortical activation in several of the ROIs, including the inferior frontal gyrus (IFG), the dorsolateral prefrontal cortex, frontal eye fields, extrastriate regions, parietal regions, and the temporal region. During the presentation of conjoined active sentences, the extra-verb violation elicited more activation in pars triangularis compared to the noun-verb agreement violation, and the opposite is true of pars opercularis, as shown in Fig. 3. During the presentation of object-relative sentences, no differences in either region of Broca's area was observed, as shown in Fig. 4.

\subsubsection{Overall results}

Several ANOVAs were computed. The first used sentence type (object-relative vs. conjoined active), type of violation (extra-verb vs. noun-verb agreement), and laterality (left vs. right hemisphere) as within subject variables. Object-relative sentences produced reliably more activation than conjoined actives $[F(1,12)=5.25, P<0.05]$ indicating the sensitivity of fMRI to the differential complexity of the two sentence types, even in a gram- 


\section{$\mathrm{z}=85 \mathrm{~mm} \quad \mathrm{z}=70 \mathrm{~mm}$}

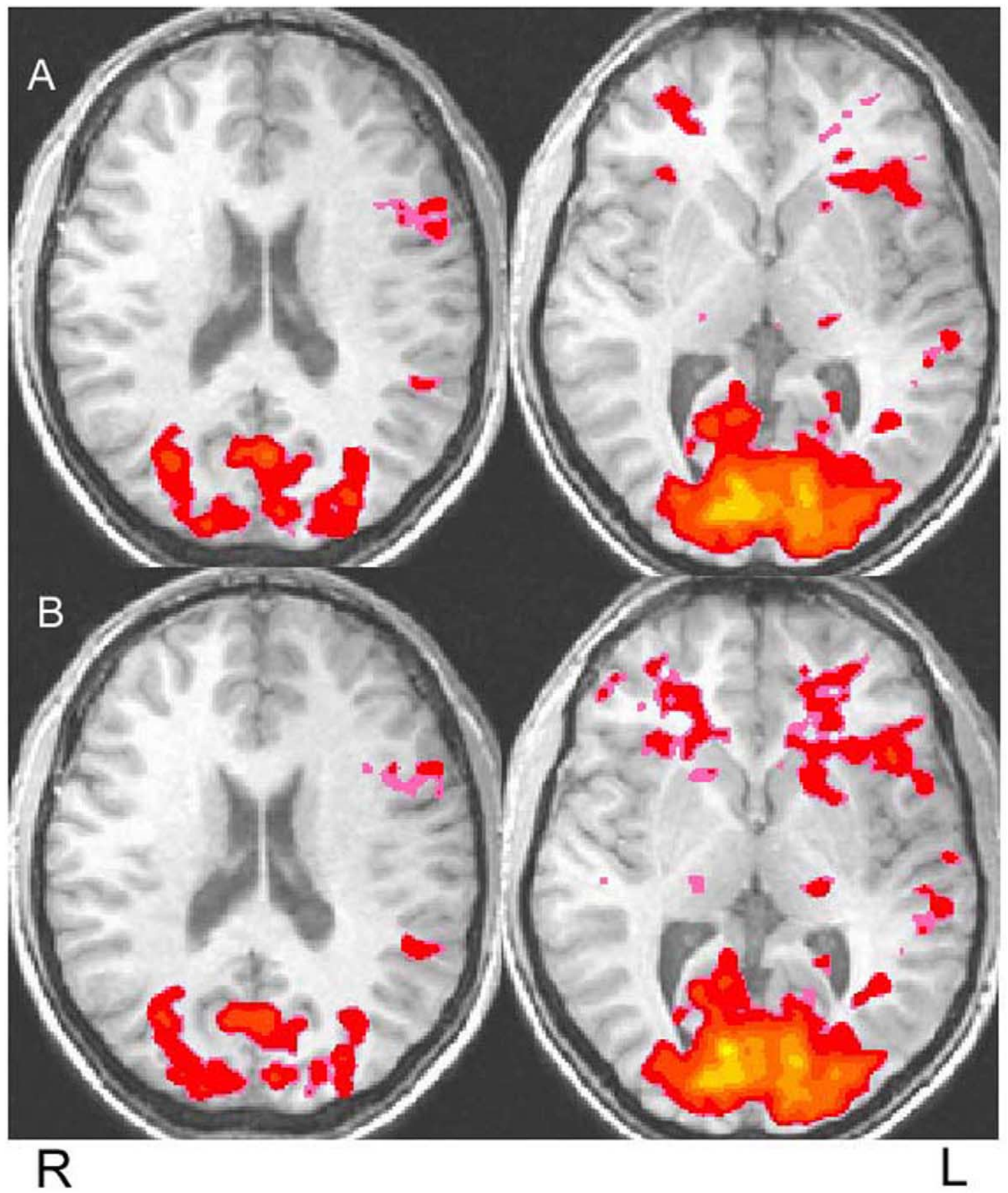

Fig. 3. The group probability maps from four axial slices depicting the activation within the two subregions of Broca's area and the temporal cortex during the processing of conjoined active sentences. The top row: (A) shows the activation elicited by the noun-verb agreement condition and (B) shows the extra-verb condition. Pars opercularis, which is located more superiorly in the brain, is shown on the left and pars triangularis, which is located more inferiorly in the brain, is shown in the right.

maticality judgment task. There was no significant effect of type of violation, $F<1$.

Separate ANOVAs were also computed for each of the ROIs with sentence type and type of violation as within subject variables. Several regions revealed significant effects of sentence type including extrastriate, parietal, and temporal cortex, along with the frontal eye fields and the opercular region of the inferior frontal gyrus, as shown in Table 1. Unlike pars opercularis, pars triangularis failed to reveal an effect of sentence type. The role of the intraparietal sulcus in language processing has not been established. However, the current study revealed substan- 


\section{$\mathrm{z}=85 \mathrm{~mm}$}

\section{$\mathrm{z}=70 \mathrm{~mm}$}

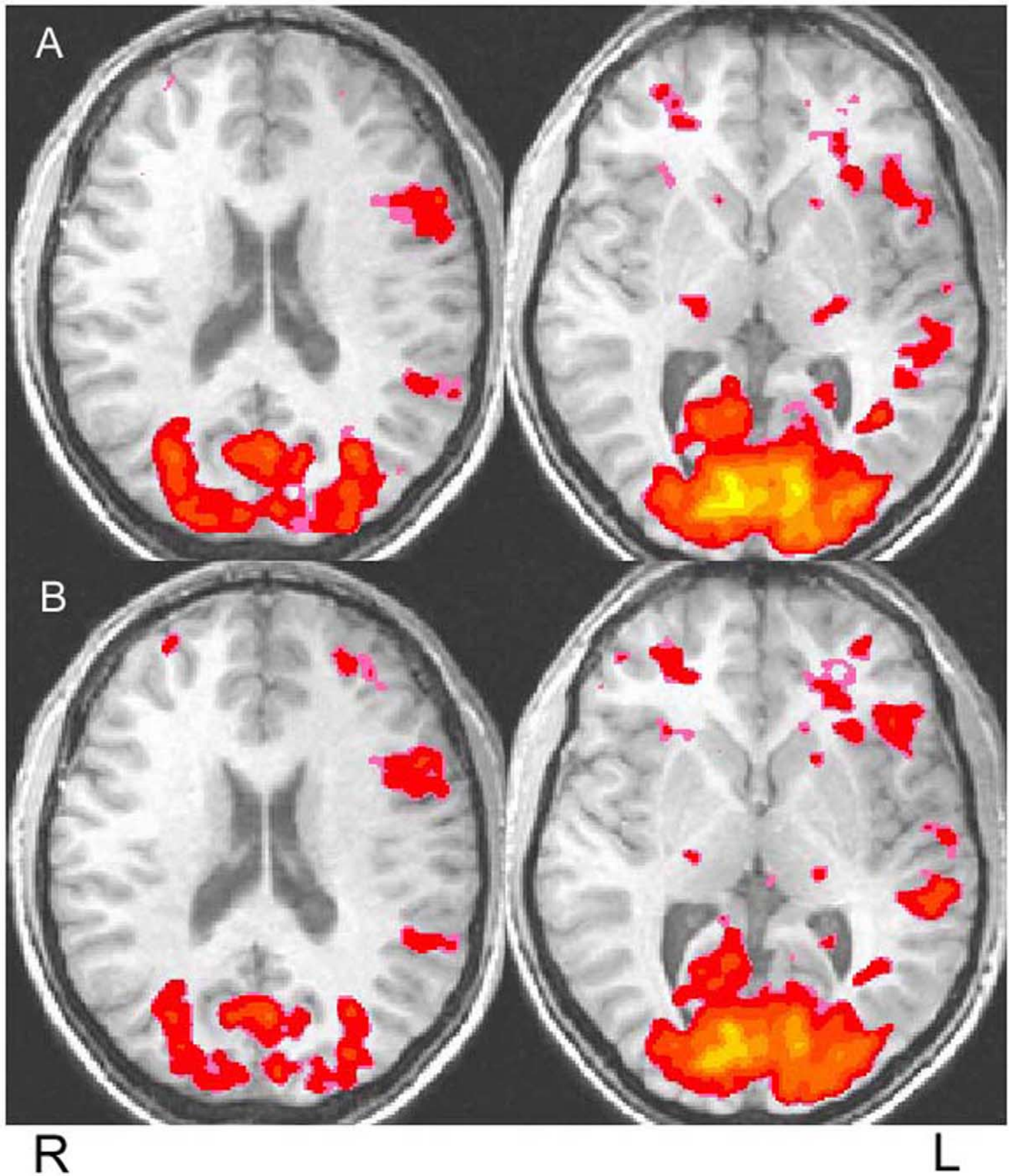

Fig. 4. The group probability maps from four axial slices depicting the activation within the two subregions of Broca's area and the temporal cortex during the processing of object-relative sentences. The top row: (A) shows the activation elicited by the noun-verb agreement condition and (B) shows the extra-verb condition. Pars opercularis, which is located more superiorly, is shown on the left and pars triangularis, which is located more inferiorly, is shown in the right.

tial, bilateral activation in the region. The type of violation did not significantly modulate the activation of either left or right IPS, but, similarly to pars opercularis, left intraparietal salcus (IPS) did reveal a significant effect of sentence type $[F(1,12)=11.08, P<0.05]$. The following two sections report the analyses that examined the effect of type of violation within the two sentence types separately.

\subsubsection{Conjoined active sentences}

The activation within pars opercularis and pars tri- 
Table 1

Activation and centroid data

\begin{tabular}{|c|c|c|c|c|c|c|c|}
\hline \multirow[t]{3}{*}{ ROI } & \multicolumn{4}{|c|}{ Sum signal intensity } & \multicolumn{3}{|c|}{ Average coordinates } \\
\hline & \multicolumn{2}{|c|}{ Conjoined active } & \multicolumn{2}{|c|}{ Object-relative } & \multirow[t]{2}{*}{$x$} & \multirow[t]{2}{*}{$y$} & \multirow[t]{2}{*}{$z$} \\
\hline & Extra-verb & Noun-verb agree & Extra-verb & Noun-verb agree & & & \\
\hline L. Dorsolateral prefrontal cortex & 10.8 & 6.6 & 11.6 & 9.6 & -32.3 & -30.7 & 24.0 \\
\hline L. Extrastriate* & 30.6 & 31.1 & 34.1 & 36.1 & -31.9 & 61.7 & -5.5 \\
\hline L. Frontal eye fields & 12.7 & 12.3 & 19.7 & 16.3 & -35.7 & 1.3 & 44.3 \\
\hline L. Intraparietal sulcus* & 18.1 & 20.7 & 25.1 & 18.4 & -25.9 & 65.1 & 40.7 \\
\hline L. Pars opercularis* & 9.9 & 12.2 & 14.2 & 13.4 & -41.7 & -7.9 & 28.2 \\
\hline L. Temporal* & 12.2 & 9.3 & 15.7 & 12.0 & -49.0 & 30.0 & 6.1 \\
\hline L. Pars triangularis & $6.1^{\dagger}$ & $3.9^{\dagger}$ & 5.5 & 6.4 & -36.9 & -25.2 & 7.9 \\
\hline R. Frontal eye fields & 5.9 & 6.2 & 8.3 & 8.4 & 38.9 & 2.6 & 46.4 \\
\hline R. Dorsolateral prefrontal cortex* & 9.8 & 7.4 & 9.1 & 8.6 & 32.9 & -25.5 & 31.5 \\
\hline R. Extrastriate & 16.7 & 17.8 & 20.0 & 17.7 & 27.6 & 65.8 & -3.4 \\
\hline R. Intraparietal sulcus & 17.5 & 16.0 & 17.7 & 21.9 & 25.5 & 62.4 & 42.0 \\
\hline R. Pars opercularis & 2.5 & 1.7 & 2.0 & 2.7 & 42.7 & -13.7 & 30.1 \\
\hline R. Temporal & 2.5 & 1.9 & 2.7 & 1.3 & 49.3 & 18.6 & 3.1 \\
\hline R. Pars triangularis & 2.3 & 1.3 & 0.7 & 2.1 & 39.0 & -23.0 & 7.6 \\
\hline
\end{tabular}

* Indicates significant effect of sentence type, $P<0.05$.

${ }^{\dagger}$ Indicates significant effect of violation type, $P>0.05$.

angularis appears to be complementary during the processing of conjoined active sentences. Pars opercularis exhibits more activation during the noun-verb agreement condition compared to the extra-verb condition; by contrast pars triangularis exhibits more during the extra-verb condition compared to the noun-verb agreement condition, as shown in Fig. 5. An ANOVA with ROI (left operculum versus left triangularis) and violation type (noun-verb agreement versus extra-verb) as within subject variables revealed a significant interaction between violation type and ROI $[F(1,12)=8.01, P<0.02]$, supporting the hypothesis that these two subregions of the IFG are functionally different. The main effects of ROI and violation type were not reliable $[F(1,12)=2.1, P>0.1 ; F<1$, respectively].

An ANOVA with type of violation as a within subject variable was computed for each of the ROIs. This analysis revealed that the conjoined active sentences elicited significant main effects of the type of violation within left pars triangularis $[F(1,12)=5.63, P<0.04]$. In addition, there were marginally significant trends within left pars opercularis $[F(1,12)=4.10, P<0.07]$ and left temporal cortex $[F(1,12)=4.02, P<0.07]$.

\subsubsection{Object-relative sentences}

As predicted, we failed to observe a differential pattern of activation as a function of type of violation during the processing of object-relative sentences. As shown in Tables 1 and Fig. 6, both subregions of Broca's area failed to reveal an effect of type of violation during the processing of object-relative sentences, as did the left intraparietal sulcal region. The left temporal region also failed to reveal a significant effect of type of violation.

\subsubsection{Laterality}

As expected, the activation in the temporal region and the two subregions of Broca's area was significantly left lateralized [temporal: $F(1,12)=5.28, P<0.05$; operculum: $F(1,12)=5.44, P<0.05$ triangularis: $F(1,12)=5.65, P<$ 0.05]. The intraparietal sulcus, unlike the frontal and temporal regions, failed to reveal a laterality effect, $F<1$ indicating that both left and right IPS was equally involved in the task although their specific functions may be different.

\section{Discussion}

The new findings reported here suggest that the two functionally separable regions within Broca's area are involved in two different aspects of sentence processing. The activation within the left temporal region appears to be affected by both semantic and syntactic processing. In addition, activation in regions not traditionally thought to be centrally involved in language processing, namely the intraparietal sulcus, the frontal eye fields and extrastriate cortex, was modulated by syntactic complexity. These results affect the current view of the neural basis of language processing.

The data suggest a refinement in the conceptualization of the role of Broca's area. The frontal pars triangularis, which revealed no reliable effects of sentence complexity, appears to be differentially involved in the semantic/ thematic aspects of comprehension. Because the lexical content of verbs is thought to determine predicate argument structure and establish thematic roles [12,38], the introduction of an extra verb in a sentence would be 

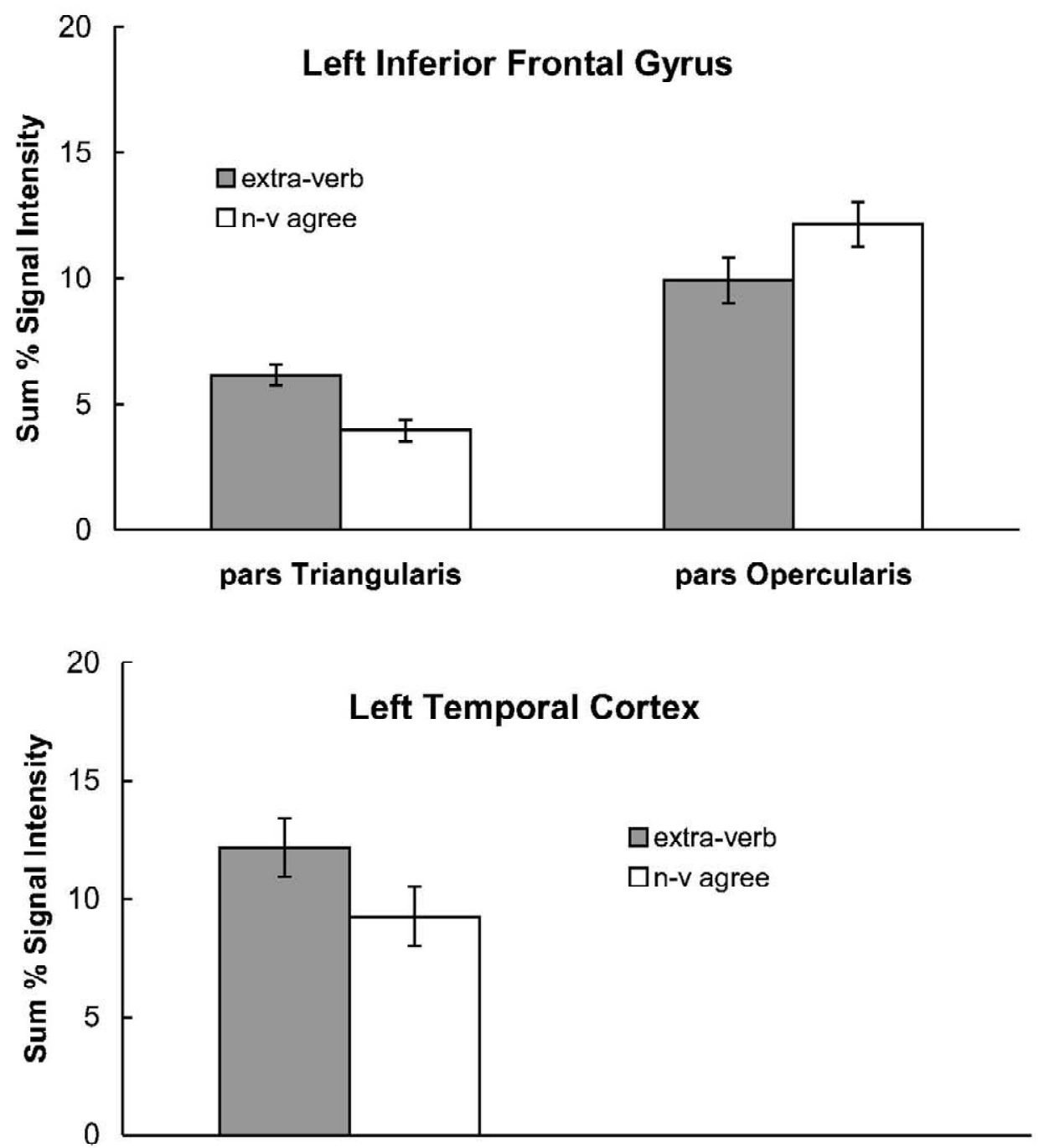

Fig. 5. The sum percent signal intensity from the left inferior frontal ROIs and the left temporal region during the processing of conjoined active sentences. Error bars represent 95\% confidence intervals based on the pooled Mse from the corresponding ANOVA (Loftus and Mason, 1994).

expected to lead to difficulties with thematic processing. Therefore, the increase in activation for the extra-verb condition in the left triangularis during the conjoined active sentences may be due to a vain attempt, at repair, to find noun phrases to which the extraneous verb can assign its thematic roles. The results suggest that the functioning of pars triangularis extends beyond semantic retrieval, and includes thematic analysis involving the verb and its arguments. Supporting the hypothesis that the pars triangularis is involved in semantic/thematic functions of language comprehension, several neuroimaging studies examining single words have found that the region is involved in semantic tasks such as verb generation [34], and semantic priming $[6,14,42,43]$. The results from these previous studies are in good agreement with the current results, which provide further support for the idea that the pars triangularis region of Broca's area is involved in semantic functions.

The frontal operculum, unlike the triangularis, revealed significant effects of sentence complexity. In addition, the region revealed more activation during the noun-verb agreement condition than the extra-verb condition during the presentation of conjoined active sentences. These results suggest that while the pars triangularis is more responsive to increased semantic demands, the operculum is more responsive to demands that require building or manipulating the syntactic structure of the sentence. In addition, a previous study found that pars opercularis was involved in a mental imagery task in which participants imagined movement trajectories [4]. These results, taken together, suggest that the frontal operculum is not exclusively involved in language processing but that the region may be involved in structure building generally.

While several studies have supported the hypothesized division of function between pars opercularis and pars triangularis, there are some studies that fail to support the division. For example, in a study by Moro et al. [29], participants read sentences consisting of pseudowords. 

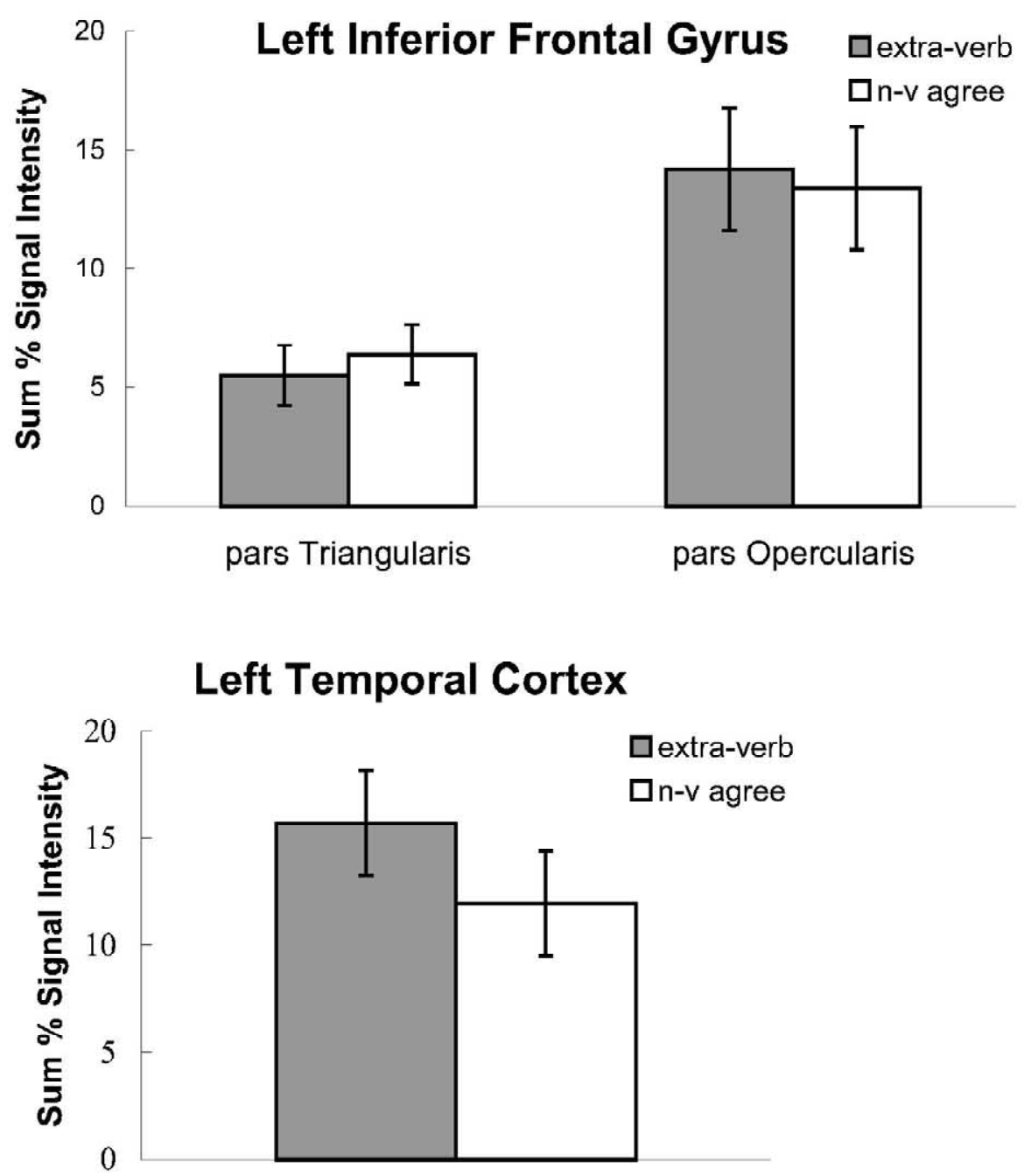

Fig. 6. The sum percent signal intensity from the left inferior frontal ROIs and the left temporal region during the processing of object-relative sentences. Error bars represent 95\% confidence intervals based on the pooled Mse from the corresponding ANOVA (Loftus and Mason, 1994).

There were three conditions, syntactic (sentences with incorrect word orderings), morphosyntactic (sentences with agreement errors), and phonotactic (sentences with words containing illegal consonant strings). Here, pars triangularis, and not pars opercularis was found to be differentially involved in processing the syntactic stimuli. In addition, pars opercularis was found to be active during all three conditions. Although the pseudowords were intended to eliminate the semantic components during reading, there is evidence to suggest that pronounceable pseudowords do, in fact, activate the semantic processing systems $[32,35]$. In addition, the incorrect word orderings may affect the semantic interpretation (or thematic role assignment) of a sentence as well as syntactic processing. Therefore, the differences in the results obtained by Moro et al. [29] and those presented in the current study may simply be a function of their unusual task demands.

The temporal region revealed significant effects of sentence complexity. As in previous studies, the region revealed a greater response to object-relative sentences than to conjoined active sentences [25,28]. In addition, there was a marginal effect of type of grammatical violation during the processing of conjoined active sentences in favor of the violation that increases the load on semantic/thematic processing, the extra-verb condition. In a study in which lexical frequency and syntactic complexity was examined, the temporal cortex was found to be differentially involved in both lexical and syntactic processing [25]. The authors suggest that the temporal region subserves "interpretive and elaborative functions involving the coactivation of distributed semantic representations which are required in lexical access, in the mapping of thematic roles and in syntactic parsing" (p. 234).

The intraparietal sulcus has long been associated with visuo-spatial processing working memory $[15,24,31,40]$. However, activation of the region, specifically in the left hemisphere, has been found in previous studies in our lab to co-modulate with the activation of the operculum in both language [31] and non-language tasks [24] and does so again in the current study. There are several possible 
explanations for the results observed in this region. One such hypothesis is that the left IPS, along with the left operculum, generates a visual image of the actions depicted in the sentence. For example, during the computation of the sentence 'Mary kicked Tom', IPS may be involved in generating a mental image of a girl kicking a boy. Another related possibility is that IPS is involved in generating a spatial structure that encodes the thematic roles of a sentence. A study of an asyntactic aphasic patient showed that he systematically used a temporalspatial strategy to map nouns onto thematic roles [12]. It was unclear whether the strategy used by this patient was a response to his linguistic deficits or if it is a normal strategy. The results presented here suggest that perhaps the use of spatial processes can occur during normal syntactic analysis and sentence comprehension. If this is indeed the case, it may account for the activation of the region during both syntactic tasks and imagery tasks.

The activation difference between the active and objectrelative sentences may provide some insight into the interaction between syntax and semantics. As stated earlier, the process of making thematic role assignments is made more difficult when the syntactic structure is complex. This was indeed observed in the differential response elicited in the pars triangularis. During the processing of conjoined active sentences, clear differences were observed as a function of violation type. This difference was attenuated during the processing of complex sentence structures, object-relative sentences. One possible explanation is that it is, by happenstance, easier to repair the extra-verb error in the object-relative condition than in the conjoined active condition. Repair in the object-relative condition can be performed by mentally inserting 'who' which co-refers to the last mentioned noun phrase. In the conjoined active condition, a pronominal subject could be mentally inserted, with the subject of the main clause as antecedent. This difference in repair may partly explain why there is no effect of violation type in the objectrelative condition, namely because both repairs are relatively easy. Further investigation of these effects and how the two violation types are dealt with on-line are necessary in order to develop a clearer characterization of these processes.

The current study required participants to make an acceptability judgment, which imposes unusual processing requirements. Grammaticality judgement tasks are thought to be easier than comprehension tasks, because comprehension requires additional semantic processing. For example, the ability of agrammatic patients to make grammaticality judgements is relatively spared despite their inability to comprehend semantically reversible sentences [2,27]. This is also observed in the finding of no significant behavioral differences in the processing of conjoined active versus object-relative sentences, a difference that has been observed several times when the task requires comprehension [25,28]. Despite this behavioral result, we did observe significant activation differences as a function of sentence type.

\section{Conclusions}

The current study provides further support for the hypothesis that there are separable functional subregions of Broca's area. The results also suggest that while the temporal cortex is affected by syntactic manipulations, it may not be responsible for syntactic analysis per se. It is difficult to separate semantic from syntactic analysis, because they apply to attributes of a sentence that are not independent of each other. Therefore it may be more useful to examine how the two types of processing demands conjointly affect the interplay of brain areas in the language network.

\section{Acknowledgements}

This work was supported by grants from the National Institute of Mental Health (NIMH-R01 MH29617, NIMHK05 MH00662, and NIMH-K05 MH00661).

\section{References}

[1] P.A. Bandettini, E.C. Wong, R.S. Hinks et al., Time course EPI of human brain function during task activation, Magn. Reson. Med. 25 (1992) 390.

[2] R.S. Berndt, A. Salasoo, C.C. Mitchum, S.E. Blumstein, The role of intonation cues in aphasic patient's performance of the grammaticality judgment task, Brain Lang. 34 (1988) 64-97.

[3] R.C. Berwick, A.S. Weinberg, The Grammatical Basis of Linguistic Performance: Language Use and Acquisition, MIT Press, Cambridge, MA, 1984.

[4] F. Binkofski, K. Amunts, P. Stephan et al., Broca's region subserves imagery of motion: A combined cytoarchitectonic and fMRI study, Hum. Brain Mapp. 11 (2000) 273-285.

[5] C.M. Brown, P. Hagoort, The processing nature of the N400: Evidence from masked priming, J. Cogn. Neurol. 5 (1993) 34-44.

[6] R.L. Buckner, W. Koutstaal, D.L. Schacter, B.R. Rosen, Functional MRI evidence for a role of frontal and inferior temporal cortex in amodal components of priming, Brain 123 (2000) 624-634.

[7] D. Caplan, N. Hildebrandt, Disorders of Syntactic Comprehension, MIT Press, Cambridge, MA, 1988.

[8] D. Caplan, N. Alpert, G. Waters, PET studies of syntactic processing with auditory sentence presentation, NeuroImage 9 (1999) 343-351.

[9] D. Caplan, N. Hildebrandt, N. Makris, Location of lesions in stroke patients with deficits in syntactic processing in sentence comprehension, Brain 119 (1996) 933-949.

[10] A. Caramazza, E.B. Zurif, Dissociation of algorithmic and heuristic processes in language comprehension: Evidence from aphasia, Brain Lang. 3 (1976) 572-582.

[11] V.S. Caviness Jr., J. Meyer, N. Makris et al., MRI-based topographic parcellation of human neocortex: An anatomically specified method with estimate of reliability, J. Cogn. Neurosci. 8 (1996) 566-587.

[12] A. Chatterjee, L.M. Maher, L.J. Gonzalez Rothi et al., Asyntactic thematic role assignment: the use of a temporal-spatial strategy, Brain Lang. 49 (1995) 125-139. 
[13] M. Dapretto, S. Bookheimer, Form and content: Dissociating syntax and semantics in sentence comprehension, Neuron 24 (1999) 427432.

[14] J. Demb, J. Desmond, A. Wagner et al., Semantic encoding and retrieval in the left inferior prefrontal cortex: a functional MRI study of task difficulty and process specificity, J. Neurosci 15 (1995) 5870-5878.

[15] V.A. Diwadkar, P.A. Carpenter, M.A. Just, Collaborative activity between parietal and dorso-lateral prefrontal cortex in dynamic spatial working memory revealed by fMRI, NeuroImage 12 (2000) 85-99.

[16] W. Eddy, M. Fitzgerald, C. Genovese et al., Functional imaging analysis software: computational olio, in: Processes in Computational Statistics, Physica, Heidelberg, 1996, pp. 39-49.

[17] D. Embick, A. Marantz, Y. Miyashita et al., A syntactic specialization for Broca's area, Proc. Natl. Acad. Sci. USA 97 (2000) 6150-6154.

[18] J.A. Fiez, Phonology, semantics, and the role of the left inferior prefrontal cortex, Hum. Brain Mapp. 5 (1997) 79-83.

[19] A.D. Friederici, B. Opitz, Y. Cramon, Segregating semantic and syntactic aspects of processing in the human brain: an fMRI investigation of different word types, Cereb. Cortex 10 (2000) $698-705$.

[20] H.D.E. Gabrieli, R.A. Poldrack, J.E. Desmond, The role of the left prefrontal cortex in language and memory, Proc. Natl. Acad. Sci. USA 95 (1998) 906-913.

[21] P.J. Holcomb, Semantic priming and stimulus degradation: Implications for the role of the N400 in language processing, Psychophysiology 30 (1993) 47-62.

[22] P. Indefrey, P. Hagoort, H. Herzog et al., Syntactic processing in left prefrontal cortex is independent of lexical meaning, NeuroImage 14 (2001) 546-555.

[23] M.A. Just, P.A. Carpenter, T.A. Keller et al., Brain activation modulated by sentence comprehension, Science 274 (1996) 114116.

[24] M.A. Just, P.A. Carpenter, M. Maguire, V. Diwadkar, S. McMains, Mental rotation of objects retrieved from memory: An fMRI study of spatial processing, J. Exp. Psychol. Gen. 130 (2001) 493-504.

[25] T.A. Keller, P.A. Carpenter, M.A. Just, The neural bases of sentence comprehension: a fMRI examination of syntactic and lexical processing, Cereb. Cortex 11 (2001) 223-237.

[26] N.A. Lazar, W.F. Eddy, C.R. Genovese et al., Statistical issues in fMRI for brain imaging, Int. Stat. Rev. 69 (2001) 105-127.

[27] M.C. Linebarger, M.F. Schwartz, E.M. Saffran, Sensitivity to grammatical structure in so-called agrammatic aphasics, Cognition 13 (1983) 361-392.

[28] E.B. Michael, T.A. Keller, P.A. Carpenter, M.A. Just, An fMRI investigation of sentence comprehension by eye and by ear: Modality fingerprints on cognitive processes, Hum. Brain. Mapp. 13 (2001) 239-252.

[29] A. Moro, M. Tettamanti, D. Perani et al., Syntax and the brain: Disentangling grammar by selective anomalies, NeuroImage 13 (2001) 110-118.

[30] T.F. Munte, H. Heinze, G.R. Mangun, Dissociation of brain activity related to syntactic and semantic aspects of language, J. Cogn. Neurosci. 5 (1993) 335-344.

[31] S.D. Newman, M.A. Just, P.A. Carpenter, The synchronization of the human cortical working memory network, NeuroImage 15 (2002) 810-822.

[32] S.D. Newman, D.B. Twieg, Differences in auditory processing of words and pseudowords: an fMRI study, Hum. Brain Mapp. 14 (2001) 39-47.

[33] L. Osterhout, P.J. Holcomb, Event-related brain potentials elicited by syntactic anomaly, J. Mem. Lang. 31 (1992) 785-806.

[34] S.E. Petersen, P.T. Fox, A.Z. Snyder et al., Activation of extrastriate and frontal cortical areas by visual words and word-like stimuli, Science 249 (1990) 1041-1044.

[35] C.J. Price, R.J.S. Wise, R.S.J. Frackowiak, Demonstrating the implicit processing of visually presented words and pseudowords, Cereb. Cortex 6 (1996) 62-70.

[36] J. Rademacher, A.M. Galaburda, D.N. Kennedy et al., Human cerebral cortex: localization, parcellation, and morphometry with magnetic resonance imaging, J. Cogn. Neurosci. 4 (1992) 352-374.

[37] F. Rosler, P. Putz, A. Friederici, A. Hahne, Event-related brain potentials while encountering semantic and syntactic constraint violations, J. Cogn. Neurosci. 5 (1993) 345-362.

[38] M. Schwartz, M. Linebarger, E. Saffran, Syntactic transparency and sentence interpretation in aphasia, Ling. Cogn. Processes 2 (1987) $85-113$.

[39] A. Sirigu, L. Cohen, T. Zalla et al., Distinct frontal regions for processing sentence syntax and story grammar, Cortex 34 (1998) $771-778$.

[40] E.E. Smith, J. Jonides, Working memory: a view from neuroimaging, Cogn. Psychol. 33 (1997) 5-42.

[41] K. Stromswold, D. Caplan, N. Alpert et al., Localization of syntactic comprehension by positron emission tomography, Brain Lang. 52 (1996) 452-473.

[42] S.L. Thompson-Schill, M. D’Esposito, G.K. Aguirre et al., Role of left inferior prefrontal cortex in retrieval of semantic knowledge: A reevaluation, Proc. Natl. Acad. Sci. USA 94 (1997) 14792-14797.

[43] A. Wagner, W. Koustaal, A. Maril et al., Semantic repetition priming for verbal and pictorial knowledge, J. Cogn. Neurosci. 9 (2000) $714-726$. 